\title{
Social Impact Assessment of the Neighborhood House (Case Study: Sixth Municipal District of Tehran)
}

\author{
Mousa Khedmatgozar Khoshdel \\ Ph. D Candidate of Sociology, University of Isfahan, Isfahan, Iran \\ Email:MKhoshdel55@Gmail.com \\ Vahid Ghasemi \\ Associate Professor in Sociology, University of Isfahan, Isfahan, Iran \\ Email:V.Ghasemi@Itr.ui.ac.ir \\ Fardin Alikhah \\ Assistant Professor in Sociology, University of Guilan, Rasht, Iran \\ Email: Faralikhah@Gmail.com
}

Doi:10.5901/mjss.2014.v5n3p580

Abstract

The study investigates citizen-centric capacities in Neighborhood Houses established in the neighborhoods of the sixth municipal district of Tehran. The study mainly aims to examine the effects of the Neighborhood House on citizens' awareness of citizenship rights and responsibilities. The subsidiary aims of the study include an assessment of the effect of Neighborhood House activities on distributive justice, increased social capital, social participation, improved quality of life, development of neighborhood identity and improved leisure patterns. According to Jürgen Habermas' discursive citizenship theory, free and equal citizens engage in dialogues in order to organize their lives not only legally but also legitimately. Social activists follow a dialogue of ideas, intentions and demands in a free, equal and fair manner. The rights and responsibilities of communicating social activists consensually emerge through dialogue rather than external factors so that citizens are committed to both their responsibilities and other citizens' rights. The methodology of the study is based on social impact assessment approach. The data was collected using both qualitative instruments such as interview and quantitative instruments such as questionnaire. Social impact assessment is the theoretical and methodological outcome of the awareness of latent, intangible functions of development plans. Attempts made to assess and estimate social outcomes, which may result from development initiatives, is called social impact assessment. The present study constitutes three stages of identification, analysis and solution. Analysis of the data obtained via interviews and questionnaires showed that the Neighborhood House initiative could assume an important role in raising citizens' awareness of their rights and responsibilities. The results showed that the important outcomes of the establishment of Neighborhood House, as a development initiative, included increased distributive justice, social participation, social capital, improved quality of life, development of neighborhood identity and improved leisure patterns, which may together help realize citizen-centric goals.

Keywords: Citizenship, Citizen-Centric, Neighborhoods House, Social Impact Assessment

\section{Introduction}

The notion of citizenship can be traced back to the Greek polis that tied rights to membership of the city, excluding women and slaves. The modern version of citizenship is connected to the twin processes of nation building and industrialization following the American and French Revolutions. Freedom of contract and protection of property rights were important elements, and the growth of markets contributed to breaking down traditional hierarchies and to fostering equality and opportunity. Citizenship has become a key concept at the center of policy debates within and across national borders. T. H. Marshall, in Citizenship and Social Class (1950), first developed a modern framework for the notion of citizenship based upon principles of freedom, equality, and solidarity. Since then citizenship has had a double focus: as a vision of equal rights and respect, and as a tool to analyze the social and political development of modern societies. (Turner, 2006: 65). 
Initially the term was used by the Greeks to denote members of that small elite within a city-state that had political rights and it was contrasted with a 'subject': someone who had a master. Until the rise of the nation-state, citizenship was either entirely absent or restricted to a very few. The modern nation-state represented an important break with earlier formations in that its legitimacy was based on its ability to embody the will and aspirations of an entire people who were in some sense all equal participants in a horizontal fellowship. This egalitarian rhetoric was eventually given substance in the expansion of the franchise until, by the early part of the 20th century, most industrial democracies gave the vote to all their members. (Bruce \& Yearley ,2006: 32-33).

Over the past decades, Tehran city has geographically developed consistent with increased population, where various cultures of different ethnicities converge. Every neighborhood has certain geographical setting where every family has specific emotions towards the location of their dwelling. Tehran municipality has considered the establishment of 'Neighborhood Houses' across the 22 municipal districts of the city in order to promote citizens' participation in social and cultural affairs consistent with neighborhood norms. Thus, the draft to establish the Neighborhood House was proposed to the City Council of Tehran, which included 8 articles of Definitions, Principles, Establishment and engagement of specialty workgroups under the supervision of the district mayor, Requirements for the appointment of the district mayor, Appointment, Responsibilities of the specialty workgroups, and Utilization of executive facilities and regulations.

Public participation initiatives developed in the Neighborhood House would serve as a bridge to improved quality of life for the citizens. A Neighborhood House is an entity within the geographical location of a municipal district, which functions under the supervision of a Board of Trustees based on certain rules and regulations. The activities assigned by the municipality and conducted by the Neighborhood House are as follows:

1. Running the entities dealing with health, sports, recreations, education, culture, religion and libraries

2. Engagement in controlling and reduction of social damages in the neighborhood

3. Identification of and supporting vulnerable groups in the neighborhood

4. Engagement in the maintenance and beautification of the neighborhood

5. Management and organization of crisis management across the neighborhood

6. Engagement in the maintenance and utilization of the monuments in the neighborhood

7. Overseeing the implementation of neighborhood development initiatives as well as needs analysis and feasibility study of various activities

8. Engagement and cooperation in education and consciousness raising in the citizens

The Neighborhood House may develop various programs based on the neighborhood capacities. Such programs may include building business, increasing public participation and development of a neighborhood identity (Sedigh, 2010:9). The Neighborhood House was an attempt to engage the citizens in managing their own affairs and consequently to devolve neighborhood-related decision-making to the residents.

One of the problems of urban management is lack of a specific framework to develop citizen participation. Recently, there have been changes in the principles of urban management, which is leant toward public involvement. Nowadays, public engagement in the management of social issues is highly emphasized. There is consensus on people's right to decide their own fates and to engage in all issues related to their social life.

People's participation in urban management can guarantee urban plans. Of the main benefits of citizen participation, besides providing information, is understanding their attitudes toward and recommendations for improved quality of urban life. Unless development initiatives pay heed to citizen needs, they fail to thrive and gain public support. Voluntary citizen participation in the urban life is associated with the following dimensions:

- Voluntary cooperation of citizens in different neighborhoods in setting public plans in place

- Citizen engagement in the design, implementation and evaluation of plans and enjoying their benefits

- An active process where the citizens, as determined and knowledgeable stakeholders, affect the outcome of plans and reach individual, group and collective growth (Vaez Mahdavi et al., 2008:17).

Citizen participation in the Neighborhood House contributes to the improvement of the neighborhood. Experience has shown that establishing 'Health House' and 'Entrepreneurship House' by using citizens' assets, beautification of unpleasant spaces through landscaping in cooperation with the residents, engagement in cultural activities including prevention of social damages such as violence, suicide and crime play a positive role in reaching social sustainability based on cooperative services in the Neighborhood House (Mansouri \& Ahmadi, 2010:4). 


\section{Literature Review and Theoretical Framework}

The concept of urban neighborhood, at the first glance, is reminiscent of a complex geographical area within the urban spaces network, which is primarily used for residential purposes followed by office, business and services uses. The sociological reality of the neighborhood is even more complex, though. According to sociologists, the traditional indicators of neighborhood specially focus on the traditional forms of villages and so on. Thus, many cities focus on professional neighborhood entities with favorable organizational structure (Rabbani, 2003:154).

Focus on human (not as the tool for but as the goal of development), children and adolescents (releasing them from discrimination and exploitation), environment, women (whose participation is necessary for development), culture, education, knowledge, ethics, security and good urban participation and governance are considered as the components of sustainable urban development (Navabakhsh \& Arjmand Siahpoush, 2009).

Social, environmental, human and economic sustainability are the major characteristics of urban development initiatives. The criteria of social sustainability include the fulfillment of needs, overcoming difficulties, encouraging and reinforcing social responsibility, developing social capital, fair distribution of opportunities, enhancing social tolerance and citizen empowerment for participation (Colantonio, 2007).

Participation is the active engagement in political, economic and cultural life as well as all other aspects of life (Taleb, 1997:556). Social participation refers to voluntary activities through which citizens participate in managing a neighborhood, city or village and engage in shaping the social life either directly or indirectly (Mohseni Tabrizi, 1990:108).

As a functional system at the micro, intermediate and macro levels, participation may contribute to social stability, increased solidarity and reduced group conflicts, developed talents and creativity, expanded democratic values and sharing power resources (Ghaffari \& Niazi, 2007:16). Participation stimulates individuals to assume responsibility in their group attempts. It may realize when indifference and irresponsibility are replaced with a sense of responsibility (Alavi Tabar, 2000:16).

Increased urban problems such as environmental contamination, traffic, overpopulation, noise pollution, expansion of informal settlements, urban instability in terms of social, economic and environmental issues, which result from unsustainable urban development, cause serious harms to the citizens such as social, mental and cultural problems. With increased problems caused by urban issues, citizenship training has gained a special niche in citizens' social and cultural development. By optimal codification of the basic components of urban training, one may increase citizen participation and reinforce desirable social citizenship behaviors. Education plays a crucial role in the development process in terms of both structural changes and individual opportunities.

In his paper 'citizen-centric city', Piran (1998) investigated the evolution of city and citizenship in Iran and across the world throughout the history. He first used the citizen-centric concept in Iran. He contended that oriental cities constituted two domains of private and governmental life where the former included family atmosphere or location and was a safe domain while outside the house was considered unsafe. He considered that occidental cities constituted three domains of private, governmental and public life, where the public domain, which belonged to citizens and was the place to nurture collective interests, was an opportunity and a place for collective decision-making. In the public domain, even the trivial issues had to be decided by consulting with and getting the agreement of the neighborhood residents. In this demarcation, public and private domains converge so much as one joins and entails the other and the city gains an internal unity so that the citizens consider the city as belonging to them and try to preserve and develop it. He eventually considered such initiatives as 'school mayor', 'city council' and 'neighborhood council' as the steps towards a citizencentric city.

In a study entitled 'how to develop a citizen-centric city: an investigation of the role of participation in educational institutions and its effect on civic participation in adulthood', Amir Panahi and Ghaffari (2009) adopted an evolutionary approach and showed that how individuals would turn into mature adults whose civic engagement contributes to the maintenance, modification or transformation of the civic society that is shaped by the individuals who are developing their own civic identities. The results showed a strong, positive correlation between the evolution of civic identity and participation in collective activities at school in adolescents and youths. The results also emphasized that schooling is a vital opportunity for practicing the democratic patterns at the lower levels of social institutions and more importantly their reproduction in the inclusive socio-political domains. Thus, the individuals whose identity is developed based on civic practices are much more prepared to contribute to the establishment of a citizen-centric city comparing with those who lack participatory experiences.

Mansouri and Ahmadi (2010) conducted a study entitled 'the role of Neighborhood House and citizen participation in social sustainability and management of neighborhoods'. They reported that urban management entities may not 
prove effective enough unless they cooperate with citizens so that citizen participation is inevitable for developing management goals and social sustainability consistent with development initiatives. They observed that urban management with social sustainability goals, limitation of unilateral decision-making by urban managers, devolution of tasks and appropriate use of local potentials and neighborhood-centered plans such as Neighborhood House are suitable approaches to realizing social sustainability.

In his most recent theories, Jürgen Habermas has proposed the concept of discursive citizenship. In this regard, free and equal citizens enter into dialogues in order to organize their life not only legally but also legitimately. Habermas presents a different framework from current citizenship theories - modern, postmodern and feministic - that considers communicative rationality, discourse ethics and communicative action as the agreements to determine citizenship rights and responsibilities. In Habermas' discursive citizenship theory, social activists pursue a dialogue of ideas, intentions, demands and expectations in a free, equal and fair manner. The rights and responsibilities of communicating social activists are shaped internally and consensually but not through external factors. Therefore, citizens are ethically committed to perform their citizenship duties and help realize the rights of other citizens. Understanding and making oneself understood as well as agreement (on such understanding) are the bases of social communicative action in cultural, social, legal and political domains (Habermas, 2001).

As one of the pioneers of good urban governance theory, Brian McLaughlin believes that urban governance should be more accountable to the changing urban trends, act appropriately in dealing with urban issues, be more inclusive of society, function as part of social learning system and play a crucial role in predicting, discovering and embracing the future. These processes are highly dependent on a network of relations inside formal government organizations, their planning systems and the relation networks among the governmental organizations, society and urban systems. In order for the urban government to function effectively as the regulator, it needs to obtain information from the social system. Appropriateness, accountability and adaptation of the government depend on its relations with the society from where realities, ideas, demands and recommendations should be transferred into official government institutions. According to McLaughlin, government is a set of legal and formal institutions with a legal power while governance is a kind of process encompassing both the government and society (McLaughlin, 1973:248).

In order to attain good urban governance, active citizen participation is fundamental - participation through a correct relationship between government and people and transparent communication channels. In the final model of good urban governance, appropriate use of people's ideas and their full engagement in urban activities are desirable though it may not become viable unless steps are taken toward participation from apparent public involvement in urban activities to people's control and authority. People's authority to shoulder their own responsibilities would become possible through participation and involvement in neighborhood and urban affairs cycle.

Sustainable local communities have a strong sense of locality. Their perspective includes all key social sectors such as commercial sector, disadvantaged groups, environment, civic associations, government agencies and religious organizations. They create capital by themselves and venture to develop creativity. Such societies value a healthy ecosystem, use resources appropriately and seek ways to improve their local economy actively. Participation among public and commercial sectors and private organizations as well as public debates become common, inclusive and interesting. Sustainability strategies in sustainable local communities develop based on extensive citizen participation (Institute for Sustainable Communities, 2006: 3).

\section{Research Methodology}

Social impact assessment involves the process of analysis, supervision and management of desirable and undesirable social outcomes (either positive or negative), planned events (policies, plans, designs and projects) and desirable and undesirable outcomes of every social change process. Rable Burdge considers social impact assessment as the regular analysis and prediction of potential outcomes of a plan, initiative or proposed policy in individual and social life (Fazeli \& Pakseresht, 2009:3).

Interagency Committee in the United States has defined social impact assessment as a set of research measures taken to evaluate, study and estimate social impacts resulted from planned measures, which should be carried out before the actual measures are implemented (Roche, 2008:10). This approach deals with sustainable changes in people's life. These impacts do not refer to immediate outcomes of development initiatives, though. Rather, they refer to sustainable changes occurring after the development initiative is implemented. That is, it is important to examine how the changes occur, which requires understanding the attitudes of development stakeholders as well as the social, economic and political context of the initiatives. Thus, it aims to manage, modify and predict the consequences of a social change 
occurred as a result of an initiative or program. The establishment of Neighborhood House in some municipal districts of Tehran poses the questions 'what are the positive outcomes of establishing Neighborhood House in Tehran neighborhoods?' and, 'what negative outcomes may result from this initiative?'

The present study adopted a mixed method where various research approaches and diverse techniques were employed consistent with the needs and environment. The study consists of three stages including identification, analysis and solution.

\subsection{Identification}

The first phase of research constitutes a field study conducted using interview and observation. Interviewing the authorities of the District-Six Municipality of Tehran, Deputy of Social Affairs and Deputy of Planning as well as interviewing Tehran municipality managers showed that various components should be incorporated into social impact assessment of the Neighborhood Houses in the sixth municipal district.

The neighborhood directors were interviewed across 18 neighborhoods of the sixth municipal district in order to investigate the weaknesses and strengths of Neighborhood House management. Interviewing the secretaries of CouncilPartner initiatives in every neighborhood revealed the considerable influence of the Neighborhood House and high potentials for wining citizen participation and trust. Besides, observation of the buildings purchased and equipped to accommodate the Neighborhood Houses as well as the real estate recommended for purchase and establishment of the Neighborhood Houses were the supplementary techniques of field observation in this phase of the study. Part of the research in this phase was also conducted using documentary study. The study covered both the literature on Neighborhood House and the literature on citizen participation. Maps and photos were also used to identify the research variables.

\subsection{Analysis}

Needs assessment techniques were used for the neighborhoods where the Neighborhood House was not yet established in order to assess cultural and social needs of the residents. For the neighborhoods in which the Neighborhood House was already established, evaluation method was used to examine the amount of success of the initiative. In this regard, the clients of the Neighborhood Houses were interviewed to evaluate the activities and tasks conducted by the entity. Subsequently, a questionnaire was developed to examine the subscales of the citizen-centric variable. The subscales included distributive justice, social participation, quality of life, social capital, neighborhood identity and attachment and leisure activities.

\subsection{Solution}

Using the strategic approach, tables were compiled of the Strengths, Weaknesses, Opportunities, and Threats (SWOT) across 14 areas of the Neighborhood House activities including Koran and Imams House, Book House, Culture and Arts House, Research House, Technology and Information House, Science and Life House, Sports House, Decision House, Health House, Toy House, Entrepreneurship House, Social Work House, Sustenance House, and Social Welfare and Services House. This was to represent the SWOT that could occur to stakeholders at various levels.

\section{Findings}

\subsection{Classification of identified outcomes}

\subsubsection{Distributive justice}

Establishing the Neighborhood House may play a crucial role in contributing to distributive justice because both similar functions are predicted for all Neighborhood Houses and neighborhood management and board of trustees are required to identify vulnerable families and help improve their quality of life. This variable was emphasized in interviewing both urban managers and Neighborhood House managers. Analysis of questionnaire results showed that the stakeholders of the Neighborhood House believed that the entity could help reduce social gap and provide a suitable context for the residents to enjoy the facilities offered by the Neighborhood House. The expected outcomes related to distributive justice 
are as follows:

- Empowerment of housewives

- Providing play facilities for children

- Providing identical opportunities for all residents to use Neighborhood House facilities

- Increased skills and abilities of clients

- Providing job opportunities for the trainees once they are trained at Entrepreneurship House

- Providing mental training and specialty skills for all clients in order to promote distributive justice

- Increasing life skills in all clients

- Providing consultation on different aspects of matrimonial life

- Providing consultation on curing addiction and training on prevention of addiction

- Providing students with dental check-up

- Providing more welfare and comfort for the residents

- Easy access to books and libraries

- Reducing commuting costs due to easy commute in the neighborhood and availability of educational facilities

- Conducting classes for all age groups

- Increasing employment rate through holding entrepreneurship classes

\subsubsection{Increased social participation}

One of the effective functions of the Neighborhood House is to encourage citizen participation across various domains of urban life. Citizen attendance at the Neighborhood House and participation in the activities related to urban life would lead to the emergence of knowledgeable and participative citizens because various sub-entities of the Neighborhood House across educational, social, cultural and psychological domains may significantly reinforce citizen participation in social life issues. Analysis of the data obtained through interviews with urban managers revealed that one of the main objectives of establishing the Neighborhood House in Tehran was to encourage citizen participation in managing the neighborhoods. Analysis of the survey questionnaire data also showed that the stakeholders of the Neighborhood House believed that the entity could further citizen participation. The expected outcomes pertaining to increased social participation include:

- Increased social vitality in the citizens

- Holding seminars and forums at the neighborhood level

- Increased social and cultural knowledge

- Facilitating voluntary participation

- Providing the context for effective citizen participation

- Reinforcing informal voluntary networks

- Causing concern about neighborhood problems

- Encouraging a sense of cooperation to solve the problems

- Providing the opportunity to hold neighborhood discussion forums and seminars

- Immediate voicing of the neighborhood problems by citizens

- Decentralized management and improved participation morale

- Providing the opportunity to train all students on waste sorting

- Holding citizenship training classes

- Using local capacities such as mosques

- Providing the opportunity to teach crisis management and safety

- Holding addiction curing classes for Narcotic Anonymous groups and supporting them

- Increasing citizen satisfaction with urban management

- Providing the opportunity to connect to other urban institutions

\subsubsection{Increased social capital}

Citizen attendance at the Neighborhood House programs may increase relations and trust as well as a mutual sense of concern for neighborhood problems. Such interaction reinforces the informal networks among citizens and increases the social capital in the clients of the Neighborhood House. A review of the literature on social impact assessment of the Neighborhood House in some neighborhoods revealed that increased social capital is one of the expected outcomes. 
The expected outcomes associated with increased social capital include:

- Creating an informal network of relations among citizens

- Increased interpersonal trust in citizens

- Increased trust in civic institutions among citizens

- Increased trust in the governance structure

- Increased solidarity and coherence among citizens

- Nurturing responsibility and commitment in the citizens toward the neighborhood

- Creating links among civic entities such as mosques, libraries, Council-Partner initiative and the Neighborhood House

- Increased legal knowledge of citizens

- Increased political knowledge of citizens

- Increased cultural knowledge of citizens

- Increased social knowledge of citizens

- Reinforcing formal and informal relations networks

- Increased number of clients to the Neighborhood House and enhancing communication among members as well as exchange of information and experiences

- Developing latent talents in the disadvantaged groups through making links with reference groups

- Holding local seminars to introduce superior talents in various domains in the neighborhood

\subsubsection{Developed neighborhood identity and attachment}

Neighborhood House may raise citizen awareness as to the specific activities done in the neighborhood. Interaction with the Neighborhood House, developing relationships with other residents and citizens and understanding neighborhood problems can enhance a sense of attachment to the neighborhood in the residents. This not only stimulates a sense of concern toward problems but also reinforces the morale for participation and neighborhood identity considering the existence of informal social relations networks in the Neighborhood House. A citizen-centric city and trained responsible citizens are only viable when the context is provided for citizen participation and engagement. The expected outcomes associated with developed neighborhood identity include:

- Increased sense of citizen attachment to their neighborhood

- Providing the opportunity for nurturing a local identity

- Forming local relation networks at different age groups

- Forming local cooperative groups for members

- Establishing local interest-free-loan funds in the neighborhood

- Local symbolization based on historical background

- Increased sense of security in the neighborhood residents

- Citizen appreciation of local religious circles

- Symbolization of the Neighborhood House and increased locality attachment

- The Neighborhood House call for citizen cooperation

- Setting appropriate patterns for adolescents and youths by inviting local elites

- Providing and introducing local symbols through publicity brochures and tracts

- Nurturing a sense of neighborhood attachment in the future generation

\subsubsection{Improved quality of life}

Specific functions of the Neighborhood House in terms of health and consultation as well as educational and sports activities may improve the quality of life of the neighborhood residents. For example, a call for participation in sports-forall or a public running race and participation in self-confidence classes may increase citizen satisfaction and awareness of life, hence the improvement of their quality of life. Of the immediate results of the Neighborhood House establishment is improved quality of life in the neighborhood residents. This may relate to various educational, social and cultural programs conducted by the Neighborhood House. The expected outcomes associated with improved quality of life include:

Improved mental and physical health in the clients

- Increased reading hours in the citizens 
- Increased competition among students

- Development of artistic and intellectual talents in the students

- Providing the context for developing creativity and innovation

- Training children and adolescents on group work

- Training on the effective use of computer and the Internet

- Easy access to libraries and increased reading per capita

- Providing training on marital relations

- Providing effective training on how to raise children

- Providing consultation on how to overcome depression

- Increasing citizen knowledge of healthy eating

- Increasing desire for life and nurturing positive thinking

- Increasing research opportunities for university and school students

- Increasing educational, training and learning kills

- Increasing university admission rate using libraries

- Increasing happiness and contentment in the neighborhood residents

- Decreasing social damages through causing citizen concerns toward improving the neighborhood status

\subsubsection{Improved leisure patterns}

The functions of the Neighborhood House would diversify the citizen leisure patterns in the sixth municipal district of Tehran. Besides, holding sports competitions and calls for participation in sports-for-all in cooperation with schools and families in local parks and sports salons in different occasions may significantly affect leisure patterns of the citizens in the sixth municipal district of Tehran. Moreover, life counseling and child rearing classes may provide homemakers with insights on effective leisure time management and recommend effective patterns of leisure activities to families. The expected outcomes associated with improved leisure patterns include:

- Providing children with happiness and entertainment through Toy House facilities in the Neighborhood House

- Holding sports competitions

- Facilitating citizen attendance at parks through public calls

- Proposing appropriate leisure patterns through holding training classes

- Using the potentials of neighborhood sports capacities to improve exercise time per capita in the neighborhood residents

- Providing citizens with the opportunity for participation in computer classes

- Providing citizens with the opportunity for participation in Koran classes

- Providing citizens with the opportunity for participation in language classes

- Providing citizens with the opportunity for participation in painting classes

- Providing citizens with the opportunity for participation in calligraphy classes

- Providing citizens with the opportunity for participation in entrepreneurship classes

- Providing citizens with the opportunity for meeting their peers and reference groups across culture and science domains

- Increasing life expectancy in the disabled clients

\section{Conclusion and implications}

\subsection{Optimistic scenario}

Neighborhood House development initiative falls within the scope of urban development plans in Tehran neighborhoods, which brings about more positive than negative consequences. It is expected that the establishment of the Neighborhood Houses in the sixth municipal district of Tehran would attract the residents to welcome the activities and programs offered by the entity, participate in the activities enthusiastically, enjoy social justice and contribute to the management of the neighborhoods. This active participation would lead to increased social capital and trust in the neighborhood residents. Moreover, it is normally expected that citizens can have a better quality of life through the facilities offered in the Neighborhood House. 


\subsection{Realistic scenario}

Interviewing the managers of the Neighborhood Houses in the sixth municipal district of Tehran and a review of the literature on the Neighborhood House initiative revealed that the entity has not yet find a suitable niche among the citizens as a locus of neighborhood activities. Thus, the entity is not yet warmly welcomed by the residents. For example, the citizens have not shown great interest in the programs so that the activities undertaken by the entity attracts a small number of clients. The Neighborhood House has failed to succeed in providing information and attracting attentions.

\subsection{Pessimistic scenario}

Unless the conditions set for establishment and ownership of the Neighborhood House change, citizens will not show interest in the entity, as they may not be willing to attend a crowded business location or a small space without parking lots. Profitability and self-sufficiency of the Neighborhood House may make the citizens disinterested in participating in the programs so that part of the 14 functions of the entity may be left idle. Furthermore, interest conflicts with previous stakeholders, particularly private institutes holding training classes, can create a gap between them and the Neighborhood House.

\subsection{Approaches to reinforcing positive outcomes of the Neighborhood House initiative}

1) Approaches associated with distributive justice

- Holding entrepreneurship classes and trying to create jobs for the vulnerable, disadvantaged social groups in the neighborhood

- Holding specialty workshops on youth or spouse problems to decrease family conflicts

- Holding language classes, extracurricular school classes and computer lessons for all neighborhood residents

- Holding entrepreneurship classes of handicrafts, tailoring, embroidery, painting and drawing

- Holding addiction prevention classes in cooperation with Narcotic Anonymous groups

- Identifying female-headed households and orphans and introducing them to supporting institutions

- Identifying vulnerable groups and facilitating the process of helping orphaned children consistent with distributive justice

2) Approaches associated with increased social participation

- Holding neighborhood seminars and local meetings with an emphasis on participatory ideas

- Conducting surveys of citizen opinions on how to improve the programs offered by the Neighborhood House and encouraging participation

- Building trust in citizens through providing information and calling for participation

- $\quad$ Training citizens on how to get prepared for dealing with natural disasters and using local volunteers

- Increasing information disclosure on the activities of the Neighborhood House to attract participation

- Accelerating the process of purchase, equipping and ownership of the Neighborhood House and preventing the process to become attritional

- Publishing 'Neighborhood Good Citizen' magazine and its free distribution among citizens

3) Approaches associated with increased social capital

- Providing motivational mechanisms such as rewards or 'elite citizen' in order to build up trust

- Build up trust in citizens through providing information, accurate performance reporting and voicing problems and barriers

- Engaging local elites to encourage citizens to attend the Neighborhood House

- Trying to identify all local elites and motivating them to cooperate with the Neighborhood House

- Revisiting the approaches to purchasing the Neighborhood House places in business centers so as to facilitate the commuting of citizens

4) Approaches associated with developed neighborhood identity and attachment

- Using the capacities of neighborhood religious circles to enhance neighborhood connections and attachment

- Participation in holding religious services in the neighborhood such as religious holidays with an emphasis on neighborhood characteristics 
- Using local mosque capacities to hold Koran, Interpretation and Hadith classes in the neighborhood

- Using the local library capacity to distribute local information brochures among citizens

- Holding citizenship training classes such as school mayor or neighborhood mayor to promote concerns toward the neighborhood

- Needs assessment of neighborhood citizens to use their ideas in strengthening local ties

- Providing information on how to establish the Neighborhood House in the neighborhoods still lacking the entity

5) Approaches associated with improved quality of life

- Providing the opportunity for maximum children use of the play facilities in the Toy House

- Increasing the number of psychology, success and self-confidence classes for all residents

- Publishing special issues of Health or Family Psychology leaflets in order to introduce appropriate patterns to families

- Using parks and green spaces in the neighborhood to hold sports competitions

- Offering specialty courses of lifestyle, consumption and nutrition to improve the quality of life

- Holding poetry, calligraphy, painting and music classes using eminent teachers in the neighborhood

- Focusing on the issue of addiction and on how to deal with the problem through cooperation with Narcotic Anonymous groups

6) Approaches associated with improved leisure patterns

- Developing and equipping sports salons in the neighborhood and using the existing capacities

- Developing and equipping play grounds in the neighborhood and using the existing capacities

- Developing and equipping fitness clubs in the neighborhood and using the existing capacities

- Developing and equipping aerobic clubs in the neighborhood and using the existing capacities

- Developing and equipping training-sports places such as soccer or volleyball and using the existing capacities

- Developing and equipping swimming pools and saunas in the neighborhood and using the existing capacities

- Holding sports competitions in the neighborhood

- Holding public family running races in the neighborhood

The establishment of Neighborhood House was a large step toward devolution of responsibilities to the citizens. Civilization and civic society, which are integral to urban life, require knowledgable, committed, responsible and active citizens. With its large population and vast geographical distribution, Tehran requires a long-term policy for future when the citizens are the managers of their own neighborhoods and city.

Study of leisure and culture needs of the citizens living in the sixth municipal district of Tehran showed that leisure patterns have turned into sedentary, ineffective patterns such as watching television due to lack of recreational facilities. With the broad scope of 14 functions predicted for the Neighborhood Houses in all neighborhoods of the city, one may hope that these patterns would change toward suitable and effective activities. The outcomes associated with the establishment of the Neighborhood House may be predicted for short-, mid- and long-term phases. The interviews showed that the entity could help train citizens in order not to be indifferent toward their neighborhoods.

The approach of social impact assessment was used to address six major outcomes of the Neighborhood House initiative including increased distributive justice, social participation, social capital, improved quality of life, development of neighborhood identity and attachment, and improved leisure patterns, which may occur both in the short- and long-term. These variables would in turn considerably contribute to developing a citizen-centric city.

\section{References}

Alavi Tabar, A. (2000). Participation in urban management: investigating citizen participation patterns in urban management (Vol. 1). Tehran: National Municipality Organization Publications.

Amir Panahi, M.,\& Ghaffari, Gh. (2009). How to develop a citizen-centric city: an investigation of the role of participation in educational institutions and its effect on civic participation in adulthood. Urban Management Biannual Journal, 24.

Bruce ,Steve \& Yearley ,Steven (2006), Sage Dictionary of Sociology, Sage publications.

Burdge, R. J. (2004), A community Guide to Social Impact Assessment. $3^{\text {rd }}$ Edition, Social Polity Press, Middleton, Wiscosin.

Colantonio, A (2007), 'Changing Social Sustainability: An Exploratory Analysis of Its Definition, Assessment, Method, Metrics and Tools" , EIBURS Working Paper Series, Oxford Institute for Sustainable Development, Oxford Brookes University.

Fazeli, M.,\& Pakseresht, S. (2009). Pamphlet of the first training workshop on assessment of social outcomes of policies, plans and 
initiatives.

Ghaderi, S. (2000). Codes and regulations of social assessment. Headquarters of Social and Cultural Studies, Social and Cultural Deputy of Tehran Municipality.

Ghaffari, Gh.,\& Niazi, M. (2007). Sociology of participation. Tehran: Nazdik Publications.

Institute for Sustainable Communities, Definition of a Sustainable Community, Retrieved January, 2006, http://www.iscvt.org.

Habermas, J. (2001). The postnational constellation and the future of democracy. Pouladi, K. (Trans.). Tehran: Markaz Publications.

Mansouri, B.,\& Ahmadi, E. (2009). The role of Neighborhood House and citizen participation in social sustainability and management of neighborhoods. Proceedings of the First Conference on Citizenship and Neighborhood Management: Rights and Responsibilities.

Mc Laughlin (1973) Control and Planning, London: faber and faber.

Mohseni Tabrizi, A. (1990). Investigating participatory contexts for villagers and their relation with agricultural extension. Tehran: Public Participation and Extension Deputy of Jihad of Construction.

Navabakhsh, M.,\& Arjmand Siahpoush, E. (2005). Principles of sustainable urban development. Tehran: Sociologists.

Piran, P. (1998). Citizen-centric city (1-5). Journal of Political and Economic Information, 119-120, 121-122, 125-126, 131-132, 133-134.

Rabbani, R. (2003). Investigating informal settlement issues with an emphasis on socio-cultural aspects (case study on Arzanan and Darak in Isfahan). Social Sciences Quarterly, 24.

Roche, C. (2008). Project impact assessment. Chavoshian, H. (Trans.). Tehran: Akhtaran Publications.

Sedigh, L. (2010). Functional capacities of the Neighborhood Houses based on neighborhood needs. Proceedings of the First Conference on Citizenship and Neighborhood Management: Rights and Responsibilities.

Turner, Bryan (2006). The Cambridge Dictionary of Sociology, Cambridge university press.

Taleb, M. (1997). Participation as the prerequisite of intervention in urban texture. Proceedings of the Conference on Tehran Urban Textures: Ministry of Housing and Urban Development.

Vaez Mahdavi, M., et al. (2008). Handbook of citizen empowerment and urban neighborhoods to improve health. Tehran: Mehr Ravash Publications.

Vanclay, Frank (2002): 'Social Impact Assessment', In M. Tolba (ed), Responding to Global Environmental Change. (vol. 4, of Encyclopedia of Global Environmental Change, series ed.: Ted Munn), Chichester: Wiley.

Vanclay, F. (2009 a,b). Principles and Guidelines for Social Impact Assessment (SIA) in the USA. In Handbook of the First Training Workshop on Social Impact and Outcomes Assessment of Projects and Initiatives (Social Appendix). Social and Cultural Affairs Deputy of Social and Cultural Studies Office. Salarvand, A. (Trans.). Tehran: Social and Cultural Studies Office of Tehran Municipality. 